the frequency of seizures with roseola. The study corroborates the suggestion that seizures with roseola, HHV-6, and fever are not always simple in type. They are frequently prolonged, recurrent, and complex, and sometimes a manifestation of encephalitis or encephalopathy. (Progress in Pediatric Neurology II, Millichap JG, Ed, PNB Publ, 1994, pp $410,415)$. These findings further weaken the hypothesis of the so-called simple febrile seizure as a distinct disease entity.

For abstracts from the 16th annual conference on febrile convulsions held in Tokyo, Dec 18, 1993, see Fukuyama Y. Brain Dev July/Aug 1994;16:339-346. Papers included neurochemical aspects, EEG studies, and clinical, epidemiological, and treatment reports. The reputed safety and effectiveness of intermittent oral diazepam $(0.4 \mathrm{mg} / \mathrm{kg}, 3$ doses $)$ at times of fever for prevention of recurrence of febrile seizures was supported in 23 children treated at Shimane Medical University and Central Hospital, Japan.

\title{
GLUTAMATE IN PYRIDOXINE-DEPENDENT EPILEPSY
}

Cerebrospinal fluid levels of glutamate, g-aminobutyric acid, and pyridoxal-5-phosphate examined in a patient with pyridoxine dependency while on and off vitamin B6 treatment are reported from Universitat Munchen, and Universitats-Nervenklinik, Wurzburg, Germany. Seizures began at age 3 weeks. Despite phenobarbital, status epilepticus occurred at 3 months and was followed by infantile spasms and hypsarrhythmia. The addition of ACTH and vitamin B6 controlled the seizures and the EEG became normal. Seizures recurred on each of several occasions when vitamin B6 was withdrawn. CSF glutamate was elevated 200-fold, whereas GABA and PLP were normal. After vitamin B6 (5 mg/kg BW/day) was reintroduced, seizures stopped and the EEG was normal, but CSF glutamate was still elevated 10 fold. A dose of $10 \mathrm{mg} / \mathrm{kg} \mathrm{BW} /$ day vitamin B6 lowered the CSF glutamate to normal levels and controlled seizures, without apparent side-effects. At age 45 months, development was normal; the head circumference having dropped from the 25 th at birth to the 3 rd percentile at 3 months was further reduced during ACTH treatment but rebounded and grew to a 50 th percentile after vitamin B6.(Baumeister FAM, Egger J et al. Glutamate in pyridoxine-dependent epilepsy: Neurotoxic glutamate concentration in the cerebrospinal fluid and its normalization by pyridoxine. Pediatrics Sept 1994;94:318-321).

COMMENT. The authors emphasize that control of seizures alone may not suffice in treating pyridoxine dependency. In order to prevent mental retardation, it is important to adjust the dose of vitamin B6 to normalize CSF glutamate levels, but using the minimum effective dosage to avoid neuropathic side effects. Glutamate is an excitatory neurotransmitter and neurotoxin, and elevated brain concentrations in infants with pyridoxine dependency may explain frequent occurrence of psychomotor retardation despite remission of seizures with vitamin B6.

\section{HYPOCALCEMIC AND HYPOMAGNESEMIC SEIZURES}

The clinical findings and neurologic outcome of 15 newborn infants with seizures due to hypocalcemia (HC) and hypomagnesemia (HM) admitted to St Louis Children's Hospital are reported from Washington University, St Louis. Patients with perinatal asphyxia, cerebral hemorrhage, or other cerebral lesion were excluded. Seven infants had associated congenital heart 
disease, 2 were premature and 2 had hypoparathyroidism. None had nutritional abnormalities. Five died and 2 had neurologic abnormalities at follow-up. Prognosis was related more to associated medical conditions than the seizures. (Lynch BJ, Rust RS. Natural history and outcome of neonatal hypocalcemic and hypomagnesemic seizures. Pediatr Neurol July 1994;11:2327). (Respond: Dr Rust, Dept Neurology, University of Wisconsin-Madison Medical School, H6/571 Clinical Science Center, 600 Highland Ave, Madison, WI 53792).

COMMENT. The associated congenital heart disease in almost $50 \%$ of these patients is remarkable and indicates the need for careful monitoring of serum calcium and magnesium levels before and after open heart surgery. Although hypocalcemic seizures caused by high phosphate content of cow's milk-based infant formula is now a rare event, seizures related to low calcium and magnesium may be experienced in infants with parathyroid disorders, low birth weight, prematurity, fatty diarrhea, gastrointestinal disease, chronic nephropathy, and cardiac disease, in addition to infants with asphyxia, brain hemorrhage, and other cerebral lesions. In four infants with hypocalcemia treated personally at the Mayo Clinic, a history of cerebral anoxia or intracranial hemorrhage was reported at birth, and in one infant, an intracranial tumor was diagnosed. Seizures did not respond to intravenous calcium gluconate and a neurogenic hypocalcemia was postulated. (Millichap JG. Nutrition, Diet, and Child's Behavior. Springfield, CC Thomas, 1986).

\section{PROLONGED QT SYNDROME PRESENTING AS EPILEPSY}

Two patients, ages 19 and 12 years, and a review of 8 previous cases of prolonged QT syndrome presenting as epilepsy are reported from the Department of Neurology and Hospital for Joint Diseases Epilepsy Center, New York University, New York. The 19 year old woman presented with "blackout spells" at 13 years. Some were a transient light-headedness and weakness, while other attacks progressed to tonic-clonic seizures with loss of consciousness. CT, EEG, ECG, and Holter monitor were normal. Echocardiogram showed mitral valve prolapse. Carbamazepine was ineffective, and within 2 months she was found dead at home. Review of ECGs revealed a prolonged QT interval, previously unrecognized. The 12 year old boy was referred with intractable seizures since 4 years of age. He also had "panic attacks" with tachycardia, dyspnea, palm sweating, and limpness. Numerous AEDs were without benefit. EEG and ECG were normal. A Holter monitor revealed ventricular tachycardia and prolonged QT syndrome. Of a total of 10 patients, 5 had family histories consistent with congenital prolonged QT syndrome (ie. sudden death or deafness). The first convulsion occurred at an average age of 4 years. Time to diagnosis ranged from 1 to 28 years. Presyncope and "lifelessness" prior to seizures were common complaints. The beta blocker, propranolol, the mainstay of therapy, is successful in most patients. Some require a pacemaker or implantable cardiac defibrillator. (Pacia SV et al. The prolonged QT syndrome presenting as epilepsy: A report of two cases and literature review. Neurology Aug 1994;44:1408-1410). (Reprints: Dr Steven Pacia, Dept of Neurology, Hospital for Joint Diseases, 301 East 17 th St, New York, NY 10003).

COMMENT. Diagnosis of this life-threatening condition may be difficult, but several factors should alert the neurologist to the cardiac origin of the seizures: 1) history of tachycardia, and presyncopal or lifeless 